\title{
Ethics in Medicine
}

\section{Research ethical committees in Scotland}

\author{
IAN E THOMPSON, KATE FRENCH, KATH M MELIA, KENNETH M BOYD, \\ ALAN A TEMPLETON, BRIAN POTTER
}

\begin{abstract}
A questionnaire was sent to 41 ethical committees in Scotland requesting information about their constitution and supervision of clinical research. Thirty-four $(83 \%)$ replies were received. Committees varied in size from one to 73 members, most of whom were medical. Ten had no nurse members and only three had lay members without direct NHS connections. Sixteen saw their role as advisory rather than supervisory. Thirteen had not met in the past year whereas two had held 10 or more meetings. Limited use (12) was made of standard protocols, and only six had formal procedures for monitoring research in progress. Only seven of 370 proposals were rejected outright. Risk/benefit dilemmas and difficulties relating to informed consent were the commonest problems encountered. The committees provide only limited safeguards for patients and research workers, and more effective, standardised procedures are indicated.
\end{abstract}

\section{Introduction}

Questions about the nature, powers, and functioning of research ethical committees have arisen in the course of several research studies conducted over the past five years by the Edinburgh Medical Group Research Project in Medical Ethics and Education. ${ }^{12}$ Disquiet has been expressed from two opposite quarters. On the one hand, research workers are concerned that the progress of medical research is being impeded by overcautious ethical committees. The controversy provoked by a study $^{3}$ of the prevention of neural-tube defects provides a recent example. On the other hand, press, television, and some clinicians ${ }^{4}$ have questioned the accountability of research ethical committees and their abilty to safeguard the interests of patients. ${ }^{5-7}$

The need for informed public debate about the supervision of

$\overline{\text { Edinburgh Medical Group Research Project in Medical Ethics and }}$ Education, University of Edinburgh

IAN E THOMPSON, PHD, EMG research fellow

KATE FRENCH, BA, EMG research assistant

KENNETH M BOYD, PHD, EMG research fellow

Nursing Studies Research Unit, University of Edinburgh

KATH M MELIA, BNURS, research associate and nursing adviser

Department of Obstetrics and Gynaecology, University of Edinburgh ALAN A TEMPLETON, MRCOG, lecturer, medical adviser

Regional Accident Unit, Royal Infirmary, Edinburgh

BRIAN POTTER, MB, CHB, senior registrar, medical adviser clinical research is underlined by the fact that large sums of public money are spent on medical research. From a total budget of about $£ 62 \mathrm{~m}$, the Medical Research Council (MRC) disbursed some $£ 52 \mathrm{~m}$ on clinically related research in $1978-9 .{ }^{8}$ In awarding grants the MRC stipulates that: "Local ethical committee approval is required for research that involves clinical trials and for investigations involving human subjects, and appropriate evidence of such approval must be incorporated in the application. ${ }^{9}$

Little has been written about the functioning of research ethical committees in Britain. ${ }^{10} 11$ We have therefore carried out a survey of Scottish committees before undertaking a nationwide survey to collect information about the nature, scope, and mode of operation of all existing research ethical committees.

\section{Methods}

A postal questionnaire consisting mainly of precoded, fixed-choice questions, with a few open-ended questions was devised. Five committees (one area, two district, one hospital, and one based on a clinical division) assisted in a pilot study, and changes were made in the questionnaires as a result. Working from a list provided by the Chief Scientist Office, based on a survey made in 1977 by the Scottish Home and Health Department, postal questionnaires were sent in January 1980 to all 43 committees. In the course of the survey three further committees came to light and these too were sent questionnaires. Five committees were found not to exist.

Thirty-four $(83 \%)$ replies were received, and further information was sought about the seven non-respondents. Two objected to questionnaire-based research but admitted that the state of their records did not permit them to complete the factual questions. Three district ethical committees were each effectively administered by one man. In one case ethical matters were "simply dealt with by the divisional clinical committee" and no separate records existed. One committee, based on a clinical division, replied that it had decided to review its whole procedure and to seek new guidelines before completing the questionnaire. One area committee had met only once.

Completed questionnaires were coded and then processed on the ICL 2980 computer at the Edinburgh Regional Computing Centre.

\section{Results}

The 34 committees were based on area (11), district (10), hospitals (five), and clinical divisions (eight).

REMIT

While all were ostensibly set up in the light of the 1964 MRC statement "On responsibility in investigations on human subjects"12 and recommendations contained in the $1967 / 73$ reports of the Royal College of Physicians of London, ${ }^{13}$ there was considerable variation in the way they interpreted their remit.

The Scottish Home and Health Department memorandum 30/197214 
laid down that hospital authorities have a responsibility to ensure that research investigations "are subject to appropriate scrutiny," and that supervisory committees should be set up, "to examine and approve clinical research investigations so that the public, the hospital and the investigator are equally protected." Ten committees saw their function as "examining" and "vetting," 10 "to consider" and "comment," and six as "merely advisory." Eight enclosed copies of the SHHD memorandum without specifying what they did, and three did not know or were unsure of their function. Only 11 considered legal as well as ethical matters. Direct supervision, monitoring, or control of research were not mentioned.

\section{COMPOSITION}

Committees ranged in size from one to 73 members, but 21 had seven or fewer full voting members. The number of doctors ranged from one to 57, with 25 having up to seven doctors. Ten committees had no nurse members, 18 had one, and the remainder two to four nurses; only five committees had nurses with any training in research. Of the 23 committees with non-medical members, 19 had either fulltime or part-time NHS employees (such as health board chairmen); 12 had members with legal or chaplaincy backgrounds. Terms of office ranged from a year to an indefinite period, with only five having any members specified as holding office for less than three years.

Committees had various methods of selection for different groups within the membership. Doctors might be members ex officio, while the nurse or lay member might be selected or nominated. In 23 committees there was selection by higher authority or other committee, in six election, in nine some membership was ex officio, and in two all medical and research staff were full members. Asked to specify criteria for selection of members, 19 committees mentioned either professional qualifications or membership of an approved professional group. Only seven mentioned interest in or experience of research, and three interest in or knowledge of ethics.

\section{MODE OF OPERATION}

The number of meetings varied considerably: two committees held 10 or more meetings a year, while 13 had not met in the previous 12 months; 11 met three or fewer times a year. Seventeen committees conducted their business informally by letter or telephone. Only 12 had a standard protocol for the submission of research proposals, and nine of these had drafted their own protocol and made this available to research workers.

Nine committees had considered no research proposals during the past 12 months. Twelve had considered four or fewer proposals and six 20 or more (one committee considered no less than 71 research proposals in a year). Predictably the bulk of research proposals came from the four centres with medical schools. The time between submission of a proposal and a decision was less than a month for 18 committees, the rest giving an initial decision within three months. Only seven of 370 proposals considered in the past year were rejected outright, and these were by four committees. A further 53 were returned for modification; 46 were resubmitted and all but one accepted. Fourteen proposals were initially rejected on ethical grounds, one because of bad research design, and two on both grounds. Other reasons for turning down research proposals were not specified. There was no relation between the number of proposals considered and the number of meetings held: one committee dealt with 71 proposals in two meetings, while another considered 39 in the course of nine meetings.

Fourteen committees had procedures whereby third parties-for example, hospital pharmacists-could lodge objections to research projects, either formally (six) or by informal communications; half usually or sometimes made use of outside assessors. Oniy three committees had ever appealed to a higher ethical committee for advice, and no appeals had been made by disappointed applicants to a higher body. Fourteen committees required a member to absent himself from a meeting if a research proposal of his own was being considered and 12 did not. No committee invariably monitored research and 19 never did so; only six had formal procedures for monitoring. Progress reports were requested regularly by three, sometimes by one, and never by 15, while only seven requested a final report from the research worker on completion. Fourteen committees did not know whether the projects they approved ever got started.

\section{ETHICAL PROBLEMS}

Asked to specify the ethical problems faced by committees, two replied only "routine," while a further four said the question was not applicable. Four of the remainder said that they had no problems, but the multiple ethical aspects of a research project encountered by these 28 committees are illustrated in the table.

Ethical problems encountered by 28 committees

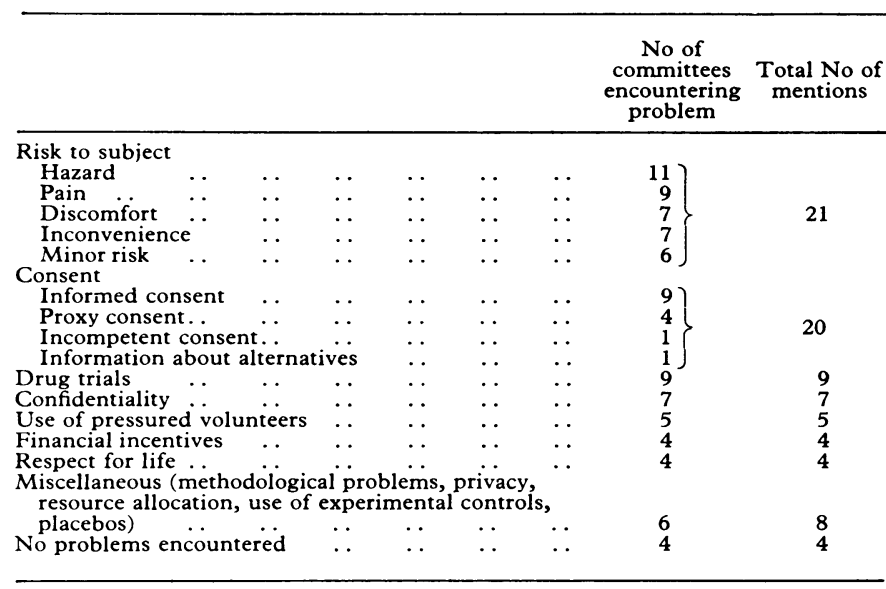

\section{ASSESSMENT}

All committees were either entirely (12) or largely (19) satisfied that they ensured the maintenance of proper ethical standards in medical research, but three were not altogether certain that the interests of patients were safeguarded. Twenty-four were quite sure that the cause of medical research was not impeded by vetting procedures, but half the committees believed that the interests of researchers were not always met. Only one committee wanted greater powers to monitor research in progress.

\section{ACCOUNTABILITY}

Sixteen committees were answerable to one other group, nine to three groups, and one to as many as eight; six were accountable to a multiplicity of medical interests. Most committees kept minutes or records of their decisions, but four did not indicate what their practice was and two did not keep any records. Nineteen regularly reported to another committee or higher authority, though this was usually another medical committee rather than a health board or lay committee.

\section{Discussion}

There is a considerable and confusing lack of uniformity in arrangements for the different types of ethical committees. Even at the same level they vary dramatically in size, composition, remit, and mode of operation. Some serve as little more than debating forums, while others seek to maintain quite strict supervision of research. Not only does this make accurate comparisons difficult, but it is impossible to assess whether uniform standards are maintained.

It is arguable that there is an inherent ambiguity in the original MRC guidelines and the statement by the Royal College of Physicians of London. This may explain why ethical committees appear to be uncertain whether their role is purely advisory or whether they are responsible for monitoring research in progress as well. While no committee mentioned supervision or monitoring of research as part of their remit, six committees had formal procedures for monitoring and seven requested a final report from the research worker. On the other hand, 14 commit- 
tees were unaware of whether approved projects ever got started. Informal communications received during the survey suggest that this ambiguity in the role or function of research ethical committees is the cause of frustration among research workers and wider scepticism that they provide adequate means for protecting the rights of patients.

Despite the suggestions in the original guidelines that ethical committees should be multidisciplinary and contain both nurse and lay members, nearly a third of all committees had no nurse member and only three had lay members from outside the NHS. The role of nurses in the practical implementation of many research projects and the role of lay members in protecting the interests of patients requires that they should be better represented on ethical committees. The excuse given by several exclusively medical committees that nurses and laymen know nothing about medical research fails to take account of the reasons for their presence. Several respondents complained that ethical committees based on a'single clinical specialty could become "closed shops," and suggested that they should include members of other specialties, representatives of other professions, and lay members.

The fact that there was no correlation between the number of meetings held by a committee and the number of research proposals it considered suggests that the rigour with which proposals are scrutinised varies considerably. It also casts doubt on the reliability of records kept and estimates given of the time taken to give initial approval to a research project.

Special difficulties arise with large projects and epidemiological studies because certain areas have no committees and adjoining districts may have different arrangements for the supervision of research. There is no certainty about which committee is responsible for supervision and co-ordination or has ultimate authority. Two respondents suggested that a national ethics committee should be set up for the approval of epidemiological studies or large-scale screening programmes, for the proper control of confidential medical records, and to serve as an advisory body or court of appeal for researchers and local ethical committees.

Among a sample of a dozen medical staff and researchers, four were unaware of the existence of their local research ethical committee. More could be done at an official level to advertise the existence of these bodies and to ensure that all clinical research investigations are submitted for approval.

In their present form research ethical committees do not satisfy fully the interests of the public or the research worker.
There is inadequate representation of lay interests at all levels, and with most committees maintaining strict confidentiality over their proceedings there is little other scope for public accountability. The limited use of expert assessors and capricious monitoring leave the research worker in a state of uncertainty.

We thank the research ethical committees that participated in this study and in particular those that also participated in the pilot survey. We also thank Professor A P M Forrest of the department of clinical surgery and Professor R V Short of the MRC Unit for Reproductive Biology in the University of Edinburgh for their help.

The Edinburgh Medical Group Research Project has been supported by grants from the Nuffield Provincial Hospitals Trust and the Leverhulme Trust Fund. The costs of the present survey were met by a grant from the Lothian Health Board.

\section{References}

${ }^{1}$ Boyd KM. The ethics of resource allocation in health care. Edinburgh: Edinburgh University Press, 1979.

2 Thompson IE. Dilemmas of dying. A study in the ethics of terminal care. Edinburgh: Edinburgh University Press, 1979

${ }^{3}$ Smithells RW, Sheppard S, Schorach CJ, et al. Possible prevention of neural-tube defects by periconceptional vitamin supplementation. Lancet $1980 ; \mathrm{i}: 339-40$.

1 Pappworth MH. Medical ethical committees. A review of their functions. World Medicine 1978;13:19-78 (seriatim).

5 Veatch RM. Hospital ethics committee: Is there a role? Hastings Cent Rep $1977 ; 7: 22-5$

${ }^{6}$ Levine C. Hospital ethics committees: A guarded prognosis. Hastings Cent Rep 1977;7:25-7.

7 Robertson JA. Ten ways to improve institutional review boards. Hastings Cent Rep 1979;9:29-33.

${ }^{8}$ Medical Research Council. Annual report 1978/79. London: HMSO, $1979: 77,82$.

${ }^{9}$ Medical Research Council. Project grants. Purpose of scheme and conditions. London: Medical Research Council, 1979.

${ }^{10}$ Denham MJ, Foster A, Tyrell DAJ. Work of a district ethical committee. Br Med f 1979;ii:1042-5.

11 O'Brien M. Clinical research and its ethical control in Durham between 1974 and 1979. Public Health 1980;94:288-93.

12 Medical Research Council. Responsibility in investigations on human subjects. Br Med f 1963;iv:177-8.

13 Royal College of Physicians of London. Report of committee on the supervision of the ethics of clinical investigations in institutions. London: RCP, 1967, 1973.

14 Scottish Home and Health Department. Memorandum on the supervision of the ethics of clinical research investigations. 30/1972. Edinburgh: SHHD, 1972. (HSS/1/49, 9 May 1972.)

(Accepted 22 December 1980)
WORDS PILLS and TABLETs. Pills and tablets have one thing in common - they contain a single dose of drug and are designed to be swallowed whole. In other respects they differ. A PILL (L pilula, diminutive of $\mathrm{L}$ pila, ball) is a uniformly medicated sphere that is made by mixing a drug with inert material, the excipient, to form a mass that is plastic (in the sense of having a doughy or putty-like consistency). It is then rolled into shape and finally coated with a varnish to protect the surface. The procedure is time consuming in relation to the quantity of final product and does not lend itself to mass production. For this reason the pill has been superseded by the tablet and the capsule in medical practice, though it is still made for herbalists and homoeopaths. By contrast, the TABLET (diminutive of table, L tabula, board or plank) is in general terms "a small flat and comparatively thin piece of . . . hard material artificially shaped for some purpose; a small slab" Oxford English Dictionary; hence in a pharmaceutical sense it may be described as a small compressed mass of medicament, usually circular, and flat or biconvex. While pills are of great antiquity, tablets are of comparatively recent origin. Although a compressed tablet was patented in the middle of the nineteenth century (Brockendon 1843), they were not made in any quantity until early in the twenticth century. I venture to suggest that not one doctor in a hundred who qualified after 1945 has seen a pill. The expression "pill-rolling" has been used to describe the thumb and finger movements of Parkinsonian tremor. This is curious because since the first quarter of the nineteenth century the great majority of pills were rolled between two flat wooden surfaces specially designed for the purpose. James Parkinson in his "Essay on the Shaking Palsy" (1817) makes no mention of pill-rolling, nor does Gowers in 1888 or James Collier and W J Adie in the 1922 first and later editions of Price's Textbook of the Practice of Medicine. Gowers came fairly close in describing "the thumb and forefinger, which may move as in the act of rolling a small object between their tips." Interestingly Price described a "drum tapping" movement that well illustrates the alternating pronation-supination. "Pill-rolling" appears in KinnearWilson's monumental work (1940) but within quote-marks as though he did not quite approve, and with the comment that the term was "now in vogue." This suggests the phrase gained ground in the 1930s, when perhaps the last few pills were rolled by hand. And why have I differentiated, it may seem somewhat pedantically, between the pill and the tablet? It is the current misuse of the former term in place of the latter, at first by the laity and now even by doctors, especially those who have never seen a pill. It all began with "THE PILL" which is currently the most widely used term for an oral contraceptive. "The pill" is in fact not a pill but a tablet. However, "the pill" has caught on and "to be on the pill" can mean only one thing. It must be admitted that, with present usage, "Are you on the tablet?" would sound a bit awkward.-B J FREEDMAN. 